This item was submitted to Loughborough's Research Repository by the author.

Items in Figshare are protected by copyright, with all rights reserved, unless otherwise indicated.

\title{
A class of finite-volume models for atmospheric flows across scales
}

PLEASE CITE THE PUBLISHED VERSION

https://doi.org/10.2514/6.2018-3497

PUBLISHER

(C) American Institute of Aeronautics and Astronautics (AIAA)

VERSION

AM (Accepted Manuscript)

\section{PUBLISHER STATEMENT}

This work is made available according to the conditions of the Creative Commons Attribution-NonCommercialNoDerivatives 4.0 International (CC BY-NC-ND 4.0) licence. Full details of this licence are available at: https://creativecommons.org/licenses/by-nc-nd/4.0/

\section{LICENCE}

CC BY-NC-ND 4.0

\section{REPOSITORY RECORD}

Szmelter, Joanna, Mike Gillard, Piotr K. Smolarkiewicz, and Christian Kühnlein. 2019. "A Class of Finitevolume Models for Atmospheric Flows Across Scales”. figshare. https://hdl.handle.net/2134/33340. 


\title{
A Class of Finite-Volume Models for Atmospheric Flows Across Scales (Invited Paper)
}

\author{
Joanna Szmelter *, Mike Gillard, ${ }^{\dagger}$ \\ Wolfson School, Loughborough University, LE11 3TU, UK \\ Piotr K. Smolarkiewicz ${ }^{\ddagger}$ and Christian Kühnlein ${ }^{\S}$ \\ European Centre for Medium Range Weather Forecasts, Reading, RG2 9AX, UK
}

\begin{abstract}
The paper examines recent advancements in the class of Nonoscillatory Forward-in-Time (NFT) schemes that exploit the implicit LES (ILES) properties of Multidimensional Positive Definite Advection Transport Algorithm (MPDATA). The reported developments address both global and limited area models spanning a range of atmospheric flows, from the hydrostatic regime at planetary scale, down to mesoscale and microscale where flows are inherently nonhydrostatic. All models operate on fully unstructured (and hybrid) meshes and utilize a median dual mesh finite volume discretisation. High performance computations for global flows employ a bespoke hybrid MPI-OpenMP approach and utilise the ATLAS library. Simulations across scales-from a global baroclinic instability epitomising evolution of weather systems down to stratified orographic flows rich in turbulent phenomena due to gravity-wave breaking in dispersive media, verify the computational advancements and demonstrate the efficacy of ILES both in regularizing large scale flows at the scale of the mesh resolution and taking a role of a subgrid-scale turbulence model in simulation of turbulent flows in the LES regime.
\end{abstract}

\section{Methodology Outline}

The Earth atmosphere is essentially a weakly compressible, rotating and highly turbulent fluid under gravity, with scales of motion spanning about 10 decades from planetary scale of thousands of kilometers down to dissipation scales at a fraction of a millimetre. Within the paradigm of unstructured meshes, the current paper investigates the suitability of ILES for predicting complex flow realisations found in challenging atmospheric problems, while addressing a range of aspects of numerical accuracy and efficiency. All implicit large-eddy simulations are performed using the NFT MPDATA integrators, the truncation terms of which were shown for structured grids to provide minimal artificial viscosity sufficient to regularise numerical solutions at the grid-scale and obviate the need for explicit turbulence models.

Effective simulations of all-scale atmospheric flows-e.g., cloud-resolving global weather-involve semi-implicit integration of the non-hydrostatic compressible Euler equations under gravity on a rotating sphere, while the limited area flows can be described by the Lipps-Hemler anelastic system of the governing PDEs. A consistent framework for discrete integrations of soundproof and compressible PDEs of atmospheric dynamics [1,2] is adopted in this work. The numerical approach employs an edge-based, finite volume discretisation within the NFT framework [3] based on the unstructured-mesh MPDATA [4,5] and advanced preconditioned non-symmetric variational (Krylov-subspace) elliptic solvers [6]. The specificity of the Earth atmosphere-geometrically a thin shell— necessitates direct inversion in the vertical. In the Krylov solver adopted here, a bespoke deflation preconditioner removes the inherent stiffness due to the anisotropy of the terrestrial atmosphere.

This flexible methodology enables integration of the governing PDEs formulated on a differential manifold with generalised curvilinear coordinates, but discretised using arbitrary hybrid meshes in the computational space with a co-located data arrangement for all the prognostic variables. In consequence a spherical surface with longitude-latitude coordinates can be resolved by discretising $[0,2 \pi] \times[-0.5 \pi,+0.5 \pi]$ with a variable mesh whose image in the physical space corresponds to a quasi-uniform resolution [7]. The capability for global modelling uses the high-performance ATLAS library [8,9] which handles generation and domain decomposition of the mesh, and the required connectivities. While Atlas is responsible for the massively-parallel distributed memory management, the performance is enhanced

\footnotetext{
*Reader, Wolfson School, Loughborough University, LE11 3TU, UK.

${ }^{\dagger}$ Research Associate, Wolfson School, Loughborough University, LE11 3TU, UK.

$\doteqdot$ Professor, European Centre for Medium Range Weather Forecasts, Reading, RG2 9AX, UK.

${ }^{\S}$ Research scientist, European Centre for Medium Range Weather Forecasts, Reading, RG2 9AX, UK.
} 
at the algorithmic level using a shared-memory parallelisation. This development is aimed at architectures evolving towards exascale.

\section{Numerical Results}

\section{A. Baroclinic instability}

The baroclinic instability benchmark [10] illustrates the performance of the approach for global flows. The propagation and breaking of the baroclinic wave train is shown in Figures 1 and 2, after 10 days evolution generated from a weakly perturbed unstable-equilibrium initial state, consisting of the two planetary jets in mid-latitudes. The initially induced velocity perturbation excites the development of the instability which in time causes the wave to steepen and form overturning fronts characteristic of natural weather systems. The results are consistent with those available in the literature [5] and illustrate the effectiveness if ILES.

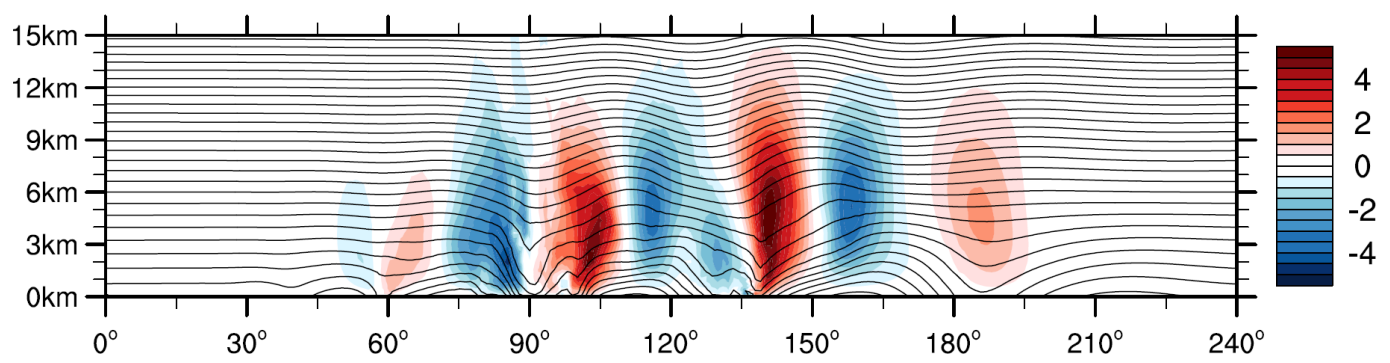

Fig. 1 Colour map of vertical velocity $[\mathrm{cm} / \mathrm{s}]$ and isolines of potential temperature in the vertical cross section $\left(53^{0} \mathrm{~N}\right.$ latitude).

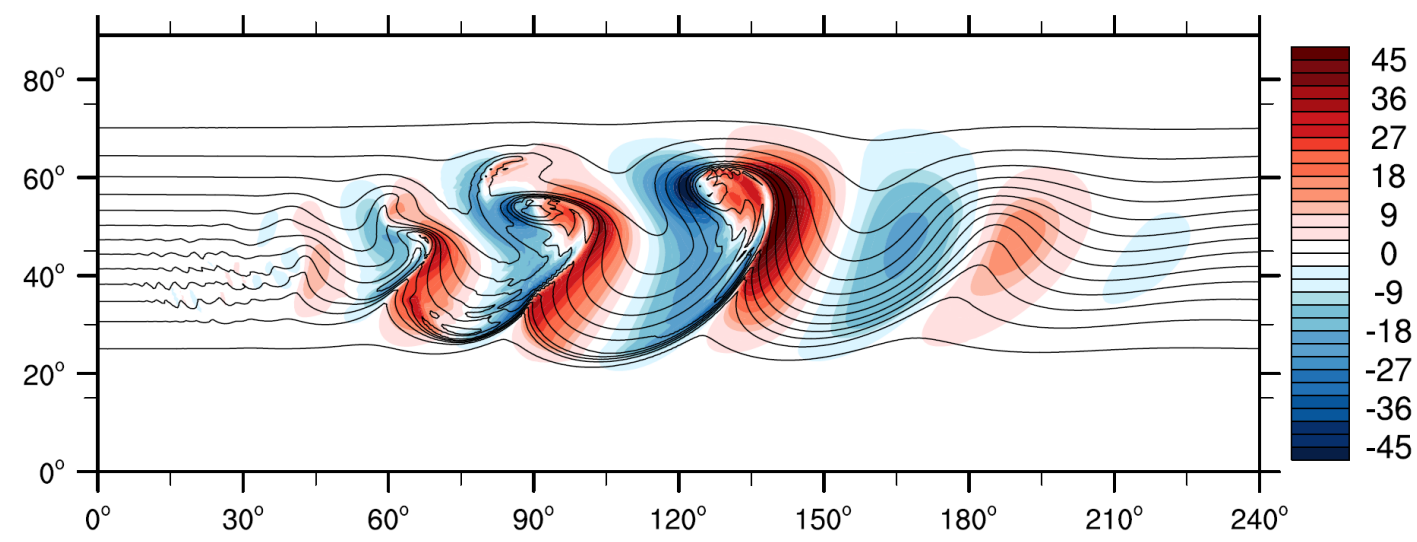

Fig. 2 Colour map of surface meridional velocity $[\mathrm{m} / \mathrm{s}]$ and and isolines of potential temperature.

The fully compressible Euler equations cast in the latitude-longitude of the geospherical framework [7] were integrated on a dual mesh created from an octahedral reduced Gaussian grid (Figure 3) with a horizontal spacing of approx $55 \mathrm{~km}$. An atmospheric depth of $45 \mathrm{~km}$ was simulated employing 30 levels with the vertical spacing increasing smoothly with height from $\delta z=175 \mathrm{~m}$ to $\delta z=3300 \mathrm{~m}$. The reduced Gaussian grid facilitates increased spacing near the poles and consequently alleviates time step limitations imposed by the stability conditions on the traditional structured lat-long grids. The treatment of periodic and pole boundary conditions follows that derived in [7]. 

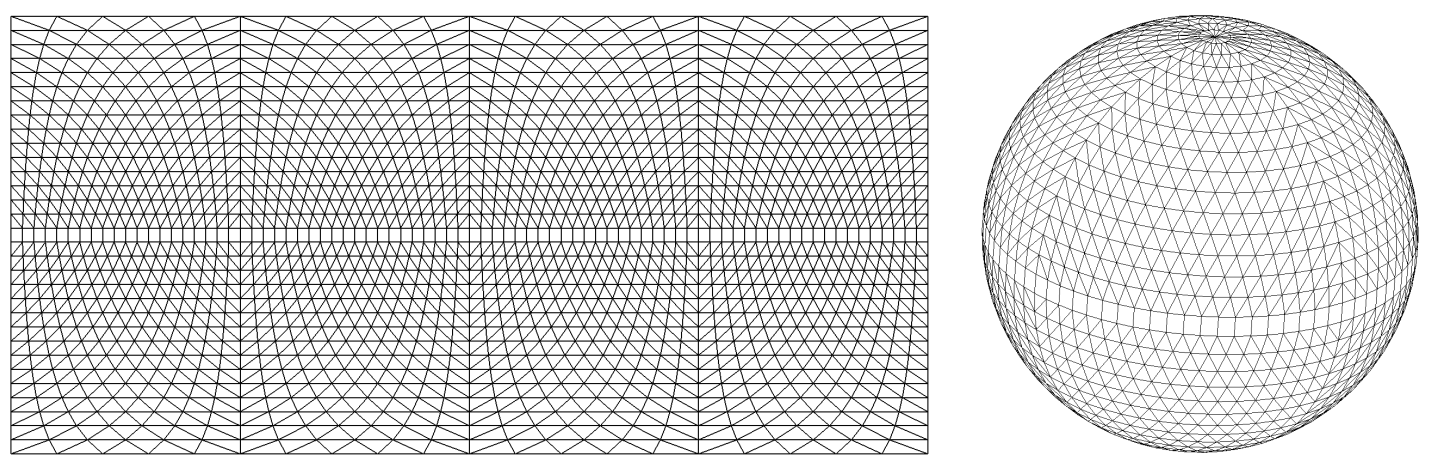

Fig. 3 Octahedral reduced Gaussian grid representation in the computational space (left) and physical space (right). A very coarse grid is shown for clarity.

\section{B. Flow past a hill}

Here, the above-described approach is used in the context of the limited area model to simulate stratified flow past a two dimensional cosine hill. The hill's half-width $\mathrm{L}$ is equal to the wavelength of the vertically propagating hydrostatic mountain wave $\lambda=2 \pi U / N$; where $\mathrm{U}$ and $\mathrm{N}$ denote, respectively, the ambient wind and buoyancy frequency. The Froude number $F r=U / N h<1$, corresponding to the dimensionless height of the hill $h / L>0.25$, was chosen to illustrate a strongly forced flow response. A two dimensional triangular primary mesh generated to conform to the cosine-shaped hill was used to construct a dual computational mesh with finite volumes of polygonal shapes. Figure 4 shows isentropes in a developing flow for the nonlinear regime where the breaking wave is apparent in the lee.

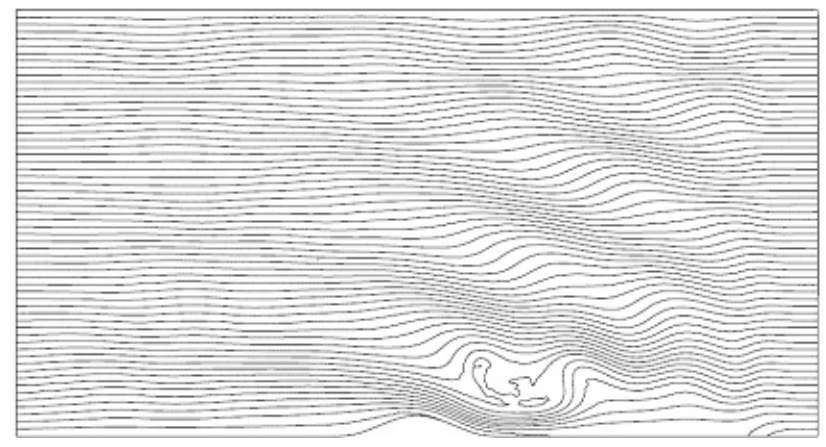

Fig. 4 Isentropes simulated using the two-dimensional limited area anelastic non-hydrostatic model; $F r<1$.

\section{Remarks}

A study demonstrating the applicability and flexibility of the finite volume discretisation operating on unstructured meshes for the NFT class of atmospheric nonhydrostatic models shows that the approach can complement established, semi-implicit semi-Lagrangian NWP methods. A record of consistent and accurate simulations using the advocated approach keeps growing $[11,12]$ and provides the evidence that the approach has the potential to efficiently resolve a broad range of atmospheric motions, from planetary down to convection where non-hydrostatic effects dominate. Further technical challenges of time to solution and energy efficiency are currently being addressed within Energy-efficient Scalable Algorithms for weather Prediction at Exascale (ESCAPE) programme of research.

\section{Acknowledgments}

This work was supported by the funding received from the European Research Council under the European Union's Seventh Framework Programme (FP7/2012/ERC Grant agreement no. 320375), and from the ESCAPE project; ESCAPE is funded by the European Commission under the Horizon 2020 Programme - grant agreement 671627. 


\section{References}

[1] P.K. Smolarkiewicz, C. Kühnlein and N.P. Wedi "A consistent framework for discrete integrations of soundproof and compressible PDEs of atmospheric dynamics". J. Comput. Phys. Vol. 263, pp. 185-205, 2014.

[2] P.K. Smolarkiewicz, W. Deconinck, M. Hamrud, C Kühnlein, G. Mozdzynski, J. Szmelter, N. Wedi, "A finite-volume module for simulating global all-scale atmospheric flows". J. Comput. Phys., Vol. 314, pp. 287-304, 2016.

[3] J. Szmelter and P.K. Smolarkiewicz, "An edge-based unstructured mesh framework for atmospheric flows". Computers and Fluids, Vol. 46. pp. 455-460, 2010.

[4] P.K. Smolarkiewicz and J. Szmelter, "MPDATA: An Edge-Based Unstructured-Grid Formulation". J. Comput. Phys. Vol. 206, pp. 624-649, 2005.

[5] C. Kühnlein and P.K. Smolarkiewicz, "An unstructured-mesh finite-volume MPDATA for compressible atmospheric dynamics".J. Comput. Phys. Vol. 334, pp. 16-30, 2017.

[6] P.K. Smolarkiewicz and J. Szmelter, "A nonhydrostatic unstructured-mesh soundproof model for simulation of internal gravity waves, Acta Geophysica". Vol. 59, pp. 1109-1134, 2011.

[7] J. Szmelter and P.K. Smolarkiewicz, "An edge-based unstructured mesh discretisation in geospherical framework".J. Comput. Phys., Vol. 229, pp. 4980-4995, 2010.

[8] W. Deconinck, M. Hamrud, C Kühnlein, G. Mozdzynski, P.K. Smolarkiewicz, J. Szmelter, N. Wedi, "Accelerating extreme-scale Numerical Weather Prediction". Chpt. in Parallel Processing and Applied Mathematics. Ed: R Wyrzykowski, Springer International Publishing, pp. 583-593, 2015.

[9] W. Deconinck, P. Bauer, M. Diamantakis, M. Hamrud, C. Kühnlein, P. Maciel, G. Mengaldo, T. Quintino, B. Raoult, P.K. Smolarkiewicz, N.P. Wedi,"Atlas : A library for numerical weather prediction and climate modelling". Computer Physics Communications, Vol. 220, pp. 188-204, 2017.

[10] P.A. Ullrich, C. Jablonowski, K.A. Reed, C. Zarzycki, P.H. Lauritzen, R.D. Nair, J. Kent, A. Verlet-Banide, Dynamical core model intercomparison project (DCMIP2016) test case document,

https://github.com/ClimateGlobalChange/DCMIP2016.

[11] P.K. Smolarkiewicz, J. Szmelter, and F. Xiao, "Simulation of all-scale atmospheric dynamics on unstructured meshes". J. Comput. Phys., Vol. 322, pp. 267-287, 2016.

[12] P.K. Smolarkiewicz, C. Kühnlein and W.W. Grabowski,"A finite-volume module for cloud-resolving simulations of global atmospheric flows". J. Comput. Phys., Vol. 341, pp. 208-229, 2017. 